\title{
Kidney Targeting and Puncturing During Percutaneous Nephrolithotomy: Recent Advances and Future Perspectives
}

\author{
Pedro L. Rodrigues, MSc, ${ }^{1,2,3}$ Nuno F. Rodrigues, PhD, 3 ,4 Jaime Fonseca, PhD,2 \\ Estevão Lima, MD, PhD, ${ }^{1,5}$ and João L. Vilaça, $\mathrm{PhD}^{1,3}$
}

\begin{abstract}
Background and Purpose: Precise needle puncture of the kidney is a challenging and essential step for successful percutaneous nephrolithotomy (PCNL). Many devices and surgical techniques have been developed to easily achieve suitable renal access. This article presents a critical review to address the methodologies and techniques for conducting kidney targeting and the puncture step during PCNL. Based on this study, research paths are also provided for PCNL procedure improvement.

Methods: Most relevant works concerning PCNL puncture were identified by a search of Medline/PubMed, ISI Web of Science, and Scopus databases from 2007 to December 2012. Two authors independently reviewed the studies.

Results: A total of 911 abstracts and 346 full-text articles were assessed and discussed; 52 were included in this review as a summary of the main contributions to kidney targeting and puncturing.

Conclusions: Multiple paths and technologic advances have been proposed in the field of urology and minimally invasive surgery to improve PCNL puncture. The most relevant contributions, however, have been provided by the application of medical imaging guidance, new surgical tools, motion tracking systems, robotics, and image processing and computer graphics. Despite the multiple research paths for PCNL puncture guidance, no widely acceptable solution has yet been reached, and it remains an active and challenging research field. Future developments should focus on real-time methods, robust and accurate algorithms, and radiation free imaging techniques.
\end{abstract}

\section{Introduction}

$\mathbf{N}$ EPHROLITHIASIS IS A SIGNIFICANT WORLDWIDE SOURCE of morbidity, ${ }^{1-3}$ constituting a common urologic disease that affects between $10 \%$ and $15 \%$ of the world population, with a subsequent clinical relapse rate of approximately $50 \%$.

Recent technologic and surgical advances have replaced the need for open surgery with less invasive procedures, such has percutaneous nephrolithotomy (PCNL) and extracorporeal shockwave lithotripsy (SWL). ${ }^{3,4}$ The selection of the specific surgical procedure usually depends on the size, composition, and location of the renal calculi, the existence of distal urinary obstructions, and anatomic variations of the urinary system. ${ }^{5-7}$

Today, PCNL is the established procedure for staghorn kidney stone removal. ${ }^{2,5,6}$ The surgery procedure usually comprises three main steps, starting with the insertion of a ureteral catheter to perform a retrograde study to evaluate the kidney anatomy and to determine whether a kidney stone is blocking the urinary tract. Then, the puncture is performed by inserting a surgical needle from the skin toward the specific calculi location. The final step concerns stone fragmentation and extraction using surgical tools, such as nephroscope, forceps, baskets, and stone lithotripters. 2,6

A worldwide study with 5803 patients was reported by de la Rosette and associates ${ }^{7}$ to assess PCNL indications, complications, and outcomes. The study reports a high success rate and a low major complication rate, showing the effectiveness and safety of minimally invasive removal of kidney stones. The most frequent complications were bleeding $(7.8 \%)$, renal pelvis perforation $(3.4 \%)$, hydrothorax $(1.8 \%)$, fever $>38.5^{\circ} \mathrm{C}(10.5 \%)$, and blood transfusion $(5.7 \%)$. Complications were assessed with Clavien classification because it provides a straightforward and validated method to classify

${ }^{1}$ ICVS/3B's - PT Government Associate Laboratory, Braga/Guimarães, Portugal.

${ }^{2}$ Algoritmi Center, School of Engineering, University of Minho, Guimarães, Portugal.

${ }^{3}$ DIGARC - Polytechnic Institute of Cávado and Ave, Barcelos, Portugal.

${ }^{4} \mathrm{HASLab} / \mathrm{INESC}$ TEC, University of Minho, Braga, Portugal.

${ }^{5}$ Department of Urology, Hospital of Braga, Braga, Portugal. 
postoperative complications. In $79.5 \%$ of cases, there were no complications, and in the remaining cases, the study presents Clavien grade I (11.1\%), II (5.3\%), IIIa (2.3\%), IIIb (1.3\%), IVa $(0.3 \%), \mathrm{IVb},(0.2 \%)$, or V $(0.03 \%)$. PCNL has also been safely applied in children with a Clavien classification comparable to those seen in adults. ${ }^{8}$ Guven and colleagues ${ }^{8}$ and Ozden and coworkers ${ }^{9}$ evaluated the safety of PCNL in 130 children with a mean age of 10.17 years and 94 children with a mean age of 9.5 years. They reported the following Clavien classification scores: Clavien grade I ( $12 \%$ vs $7.4 \%)$, II (2.8\% vs $2 \%)$, IIIa (6.4\% vs $4.3 \%$ ) (no grade IV or V were reported in either study).

The success and treatment outcomes of the surgery are very well known as being highly dependent on the precision and accuracy of the puncture step (because it must reach the calculi with a precise and direct path), making this step the most challenging task for surgeons to master. ${ }^{5,10-12}$ The ideal renal access is one that allows complete removal of the calculi while minimizing bleeding. Inaccurate needle punctures often cause complications, such as injuries in the kidney and contiguous organs, and eventually prejudice the overall surgical success and patient outcome. ${ }^{10}$

Although PCNL is considered minimal invasive surgery (MIS) with many associated benefits, such as producing small patient incisions, reducing hospitalization time, and improving postoperative recovery, ${ }^{13}$ complications may still arise. ${ }^{6}$ Among the most common complications one may find anatomic target restricted vision, difficulty in handling the surgical instruments, restrictive mobility inside the body, high dexterity levels of surgeon hand-eye coordination, needle deflections, moving anatomic target, and anatomic structure deformations and movements. 5,14

Multiple paths and technologic advances have been proposed in the field of urology and MIS to improve this procedure. In what concerns PCNL puncture in particular, the most relevant contributions have been provided by the application of medical imaging techniques, as well as the fusion of multiple imaging procedures ${ }^{10,13,15,16}$ Aside from medical imaging, robotic systems, ${ }^{13,17,18}$ navigation systems, ${ }^{19}$ finite element models, ${ }^{20,21}$ and recent developments in computer graphics and image processing ${ }^{13}$ have been proposed in recent years to improve percutaneous puncture. However, the use of new solutions not dependent on medical imaging, in current surgical practice, is still very limited.

To date, several reviews regarding PCNL have been published. All of these reviews, however, focus on specific methodologies to aid PCNL whole surgical stages, such as imaging, surgical outcomes, patient positioning, and medical instrumentation. In this study, we follow a different path, focusing solely on the puncture step of PCNL through the analysis of the most relevant techniques to its improvement, such as medical imaging, tracking systems, and robotics. Following this strategy, the main objective of this article is twofold: First, to present the state of the art in terms of methodologies and techniques for conducting PCNL puncture in today's clinical environment, and second, to provide future research paths for the PCNL procedure improvement.

\section{Methods}

Ethics approval was not needed for this review.

Analyzed articles had to be written in English and published in peer-reviewed journals or conference proceedings reporting qualitative data concerning kidney targeting and puncturing from 2007 to December 2012. No further constraints were considered. The search was subsequently updated on January 2013.

A systematic literature search was performed considering available reports at Medline/PubMed, ISI Web of Science, and Scopus databases.

Searches were performed using the following key words: "PCNL puncture," "PCNL navigation systems," "PCNL robotics," "PCNL medical imaging," "Percutaneous Robotics," and "Percutaneous Punctures." Manual search on the reference lists of the included articles were also considered.

A total of 3284 article titles were analyzed independently by two authors to determine whether the article was potentially relevant for the review topic; 911 abstracts and 346 fulltext articles were assessed and discussed independently by the same reviewers (Fig. 1). Fifty-two final reports were included in this review as a summary of the main contributions to kidney targeting and puncturing during PCNL. The remaining authors provided critical revision of the manuscript for important intellectual content and help in disagreements between article selection.

For the purpose of this review, discussed are the main benefits and limitations for kidney targeting and PCNL conduction for the following research paths: Medical imaging, surgical modifications, medical robotics, and computer-aided surgery.

\section{Results}

\section{Medical imaging for PCNL}

An overview of different advantages and disadvantages of medical imaging techniques for PCNL puncture planning and guidance is given in Table 1.

X-ray-based imaging. Fluoroscopic radiography is the most common technique for conducting the puncture in PCNL, allowing the acquisition of real-time 2D images inside the body, which provides information about the whole anatomy of the urinary system, including kidney stones. ${ }^{2}$ Fluoroscopic methods are also often combined with direct endoscopic visualization for analysis of anatomic structures. ${ }^{10}$

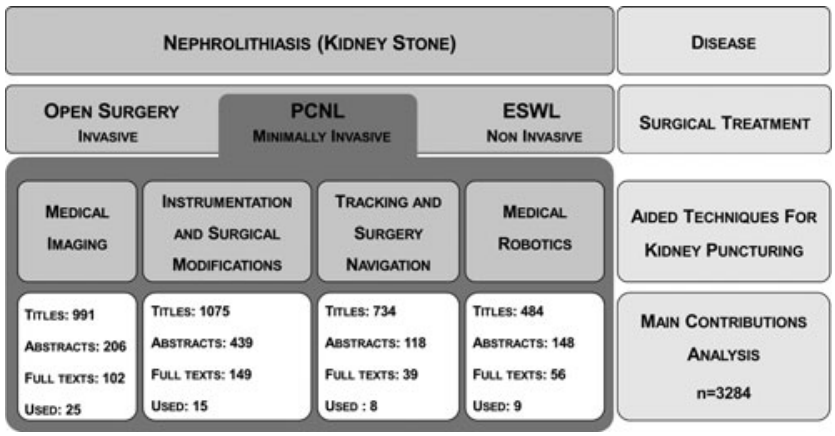

FIG. 1. Study selection process for the main contribution for percutaneous nephrolithotomy (PCNL) puncture, showing the number of titles, abstracts, and full texts analyzed for each technique for kidney puncture. ESWL=extracorporeal shockwave lithotripsy. 
Table 1. Summary of Image-Guided Techniques for Percutaneous Nephrolithotomy Puncture

\begin{tabular}{|c|c|c|c|}
\hline $\begin{array}{l}\text { Imagiology } \\
\text { technique }\end{array}$ & Advantages & Disadvantages & Main usage \\
\hline \multicolumn{4}{|c|}{ X-ray-based imaging (radiation exposure for real-time image acquisition) } \\
\hline $\mathrm{CT}$ & $\begin{array}{l}\text { - 3D reconstruction } \\
\text { - Real time }\end{array}$ & $\begin{array}{l}\text { - High cost } \\
\text { - Needs large spaces to be used } \\
\text { intraoperatively that may restrict } \\
\text { surgeon movements }\end{array}$ & $\begin{array}{l}\text { Preoperative } \\
\text { planning }\end{array}$ \\
\hline C-Arm & $\begin{array}{l}\text { - Most common technique for } \\
\text { puncture conduction } \\
\text { - Real-time images } \\
\text { - Compact and portable }\end{array}$ & - 2D images only & Intraoperative \\
\hline IVU & $\begin{array}{l}\text { - Clear visualization of anomalies } \\
\text { - Low cost }\end{array}$ & $\begin{array}{l}\text { - Needs a radiopaque dye } \\
\text { - 2D images only }\end{array}$ & $\begin{array}{l}\text { Preoperative } \\
\text { planning }\end{array}$ \\
\hline \multicolumn{4}{|l|}{ Without X-ray } \\
\hline MR & $\begin{array}{l}\text { - High contrast and resolution } \\
\text { images in orthogonal and } \\
\text { nonorthogonal planes }\end{array}$ & $\begin{array}{l}\text { - High cost } \\
\text { - Needs large spaces to be used } \\
\text { intraoperatively that may restrict } \\
\text { surgeon movements } \\
\text { - Needs nonferromagnetic surgical } \\
\text { instruments }\end{array}$ & $\begin{array}{l}\text { Preoperative } \\
\text { planning }\end{array}$ \\
\hline US 2D & $\begin{array}{l}\text { - Versatile method of conducting } \\
\text { surgical needles in percutaneous } \\
\text { procedures } \\
\text { - Real-time images } \\
\text { - Reduced cost }\end{array}$ & $\begin{array}{l}\text { - Low quality images } \\
\text { - 2D images only } \\
\text { - Image artifacts } \\
\text {-User dependence } \\
\text { - Difficulties at the identification of } \\
\text { small calculus }\end{array}$ & Intraoperative \\
\hline US 3D & $\begin{array}{l}\text { - 3D images } \\
\text { - No real-time }\end{array}$ & $\begin{array}{l}\text { - Needs experienced technicians } \\
\text { - Difficulties at the identification of } \\
\text { small calculus }\end{array}$ & $\begin{array}{l}\text { Preoperative } \\
\text { planning }\end{array}$ \\
\hline Endoscopic imaging & $\begin{array}{l}\text { - Visualization in real time of } \\
\text { adjacent structures }\end{array}$ & $\begin{array}{l}\text { - Only information about the surface } \\
\text { of organs }\end{array}$ & Intraoperative \\
\hline \multicolumn{4}{|l|}{ Multimodal imaging } \\
\hline $\begin{array}{l}\text { US \& CT } \\
\text { US \& MR }\end{array}$ & $\begin{array}{l}\text { - Combines advantages from } \\
\text { different imaging modalities }\end{array}$ & $\begin{array}{l}\text { - Needs nonrigid registration to } \\
\text { align anatomic structures }\end{array}$ & $\begin{array}{l}\text { Preoperative } \\
\text { Planning }\end{array}$ \\
\hline
\end{tabular}

$\mathrm{PCNL}=$ percutaneous nephrolithotomy; $\mathrm{CT}=$ computed tomography; $3 \mathrm{D}=$ three dimensional; $2 \mathrm{D}=$ two dimensional; IVU =intravenous urography; $\mathrm{US}=$ ultrasonography; $\mathrm{MR}=$ magnetic resonance.

The C rotational fluoroscopy (C-Arm) consists of a compact arc-shaped (180 degrees) system that is inserted under the patient stretcher. ${ }^{10,12}$ This allows the acquisition of continuous or discrete real-time images, reporting the location of kidney stones in relation to the whole surrounding anatomy. As a result of the reduced space this equipment occupies in the surgery room, percutaneous procedures and endoscopes have benefited from this method, both in planning and during the interventions. $^{10}$

Apart from fluoroscopy, CT and intravenous urography (IVU) appear as other possibilities in the field of imaging PCNL. ${ }^{22,23}$ These techniques, however, are mainly used as means of diagnosis and surgical planning, before PCNL.

IVU imaging, using a radiopaque dye, provides clear visualization of anomalies in two-dimensional (2D) images of the urinary system, thus facilitating puncture planning. As a result of its low cost and high level definition of the urinary system and kidney stones, this method has even replaced CT in some institutions. ${ }^{10,13}$

$\mathrm{CT}$, however, is the method of choice for the diagnosis and preoperative planning of PCNL, because it has a number of advantages over IVU-namely the detection of renal calculi with a sensitivity and specificity of $97 \%$ and $100 \%$, respec- tively, identification of renal lesions and blood vessels, and three-dimensional (3D) visualization of the urinary system and surrounding structures. ${ }^{10,13,24-26}$

On the other hand, CT can also be used with radiopaque contrast dye (angiographic CT) to produce detailed images of blood vessels, ureters, kidney calices, and surrounding tissues. ${ }^{10}$

Imaging without X-ray. To reduce $X$-ray use in PCNL, techniques based on ultrasonography (US), magnetic resonance (MR), and endoscopic imaging have been successfully reported in several studies. ${ }^{10,13,15}$

The use of US is being recognized as a safe and effective method of conducting surgical needles in percutaneous procedures, because it enables real-time image acquisition, is versatile, and has a reduced cost when compared with other imaging options. ${ }^{27}$ As a result of the absence of ionizing radiation, this is often the method of choice in pregnant patients and those with transplanted organs. ${ }^{10,13,28}$ Technologic advances have also enabled the acquisition of 3D images through US, providing volumetric measurements and 360degree analyses of anatomic structures.

At the level of percutaneous puncture, MR has become clinically important, because it provides images with high 
contrast and resolution, multiplanar images, and both 2D and $3 \mathrm{D}$ visualization. Furthermore, the MR allows construction of images in nonorthogonal oblique planes, which provides a complete view of the total needle length and its spatial relationship relative to the puncture target. ${ }^{10,15}$

Multimodal imaging. Several authors have explored the combination of different imaging techniques. Among these, $\mathrm{Li}$ and associates ${ }^{15}$ have explored a rigid registry process, using the Interactive Closest Points algorithm, applied to intraoperative US images and preoperative MR images, through the manual selection of pairs of points in both images from the cranial pole, caudal pole, and kidney hilum. A respiratory gating method was also used to minimize the impact of kidney deformation, where the US images were only obtained at the same stages of the respiration cycles-with a mean squared error of $3.53 \mathrm{~mm}$.

In a previous study, Leroy and colleagues ${ }^{29}$ presented an automatic rigid registration method based on cross-correlation of the intensity of US and CT images. To improve cross-correlation, the CT and US image contours were highlighted using image processing algorithms. Results present a total registration time of 80 seconds and a mean squared error of $5.1 \mathrm{~mm}$.

The rigid registration of intraoperative US images with preoperative CT volumes has been actively explored in the literature. ${ }^{22}$ Often, the use of statistical metrics, such as mutual information and correlation ratio, allow estimation of the correspondence of original CT and ultrasound intensities. Registry errors of $1.2 \mathrm{~mm}$ and target distances of $4.7 \mathrm{~mm}$ have been obtained by Mozer and coworkers. ${ }^{30}$ In this work, the authors use a $3 \mathrm{D} / 3 \mathrm{D}$ surface matching rigid registration between CT segmented structures and the 3D US images. Wein and colleagues ${ }^{22}$ presented a fully automatic image-based algorithm for registering 3D freehand ultrasound sweeps with CT. They applied a linear correlation non-rigid registration technique to achieve a processing time of 28 seconds and target distance error of $8.1 \mathrm{~mm}$.

Some of these studies also use optical tracking to display a virtual needle instrument over the registered images. ${ }^{15,22}$

Although without current application to PCNL, B-Splines have been explored as a nonrigid method, due to its simplicity and robustness, with a particular potential for alignment of lower anatomic structures. ${ }^{31}$

Endoscopic imaging. Apart from imaging methods that enable one to noninvasively visualize and collect information about internal organs and its associated phenomena, endoscope methods have gained importance in recent years for establishing a percutaneous path. ${ }^{12,13}$

Recently, Bader and coworkers ${ }^{16}$ reported a modified needle with $1.6 \mathrm{~mm}$ diameter that integrates a micro-optics of 0.9 and $0.6 \mathrm{~mm}$ in diameter. This micro-optics integrates light and can be inserted either in the puncture stylet or the working sheath of the puncture needle. This system was tested for creating percutaneous renal access in 15 patients, which resulted in a reported decrease of tract size, need of medical imaging, and morbidity. This optical needle helps the surgeon to avoid adjacent organs during puncture and to decrease the time needed to puncture. Desai and associates ${ }^{32}$ used the above needle to define microperc as a modified PCNL procedure in which renal access is performed avoiding multiple exchanges of dilators and instruments during tract dilation. Under US and/or fluoroscopic guidance, a selective caliceal puncture is made with the optical needle guidance. The authors reported that the main disadvantage was the long duration of stone fragmentation, therefore recommending it for small stones with less than $20 \mathrm{~mm}$.

This new microperc may be compared in terms of clinical outcome with flexible ureteroscopy. Ureteroscopies have been recommended as a second-line treatment for calculi with $<1 \mathrm{~cm}$, or as the third choice for stones with $1-2 \mathrm{~cm}^{6,33}$ Modern endoscopes with small diameters can be easily maneuvered into the intrarenal space from upper to lower kidney poles. ${ }^{3}$

Imaging - present and future. The medical benefits of the various imaging techniques are unquestionable for PCNL and other MIS procedures. Despite advantages on diagnosis, surgical planning, and guidance, there are some factors that limit the use of imaging techniques in renal puncture, in particular the low level of image quality, 2D imaging, and radiation exposure.

Many of the current imaging methods only allow the acquisition of 2D images (fluoroscopy, IVU, and US) and therefore do not take into account the 3D structure of the urinary system anatomy. Human error may stem from the misinterpretation of images during puncture, and fluoroscopic methods do not yet allow the distinction between different tissue layers. ${ }^{13,15,24}$

Although real-time images may facilitate the puncture step by providing a visualization of the target and surgical instruments, the use of CT and fluoroscopy are limited by the exposition of ionizing radiation and its associated risks. ${ }^{34}$ Mancini and colleagues ${ }^{35}$ reported higher body mass index, greater stone burden, nonbranched stones, and multiple nephrostomy access tracts as the main aspects for increased radiation exposure during PCNL.

Although IVU and angiographic CT have a potential to improve the image quality for puncture planning and guidance in relation to $\mathrm{CT}$, they necessitate the injection of a radiopaque dye, which can cause allergic reactions in some patients and potentially further damage kidney function, blood vessels, and nerves. ${ }^{10}$

2D US allows the acquisition of images in real-time without involving radiation. The poor image quality, shadow and speckle artifacts, and user dependence are some problems that limit its use in PCNL, however. Although the use of 3D US is a promising method for mitigating the disadvantages of 2D US, at the moment it demands experienced technicians for handling the ultrasound probe during the reconstruction of anatomic volumes. Finally, both 2D and 3D US present difficulties in the identification and visualization of the urinary system anatomy and small kidney stones, particularly in obese patients. ${ }^{4,6,15}$

The use of MR image acquisition in real time is limited by the large magnetic field generated, which disables the examination of patients with electronic devices (eg, pacemakers and defibrillators) and implies the use of nonferromagnetic surgical instruments, which increases procedure costs.

The cost and high spatial volume occupied in the operating theater by the CT and MR equipment also limits the use of these imaging methods for renal procedures, because the equipment can obstruct the surgeon's actions. 
Although multimodal imaging systems can reduce many of the problems mentioned above, the lack of algorithms for performing a robust real-time nonrigid registration continues to limit its use in MIS surgeries like PCNL. ${ }^{31,36}$

Thus, obtaining clinical relevance in the field of image recording remains a challenge and a large active research area, mainly as a result of the difficulties imposed by breathing movements and deformation of tissues during surgical manipulation. ${ }^{37}$

Finally, endoscopic methods present high spatial resolutions and provide real-time image acquisition, allowing direct visualization of the structures manipulated in surgery. The use of flexible ureteroscopy in pediatric patients has been debatable, because of the small caliber urethra and ureter. Consequently, microperc may be more valuable and with no morbidity associated with tract size. ${ }^{16}$

These methods, however, only provide information about the surface of organs and cavities, therefore preventing use in surgery planning and limiting capability for puncture guidance. $^{13,34,36,37}$

\section{Procedural improvements and medical instrumentation}

In the PCNL procedure, the final needle position influences the efficiency with which the kidney stones are fragmented and removed, because it provides the path from the skin periphery to the renal calix. ${ }^{11}$

The positioning of these percutaneous surgical needle interventions is usually held according to linear paths from the skin periphery to the anatomic target, as a result of the lack of tactile feedback and mechanical systems to control the orientation and position of the needle tip.

Inaccurate punctures can cause damage in kidneys and its surrounding tissues and organs. ${ }^{6,10}$ The learning curve for PCNL is estimated to be around 60 cases, a situation that makes complication rates increase during the long surgeon training period. ${ }^{11,38}$

The needle trajectory in puncture processes cannot be changed once the needle is inserted to a depth greater than $2 \mathrm{~cm}$, although the kidney is normally at a depth of 5 to $10 \mathrm{~cm} \cdot{ }^{14,39}$ If the needle does not hit the target, it has to be removed from the body and reinserted. Therefore, one may need to make several attempts to accomplish a correct needle insertion, consequently causing an increase in postoperative complications, poorer surgical outcomes, and increased likelihood of perforation of surrounding structures.

The leading cause for needle deflection depends on the needle diameter, perforation depth, needle tip shape, mechanical properties of the tissue, insertion force, insertion speed, and frictional forces between the needle and tissue. ${ }^{20,21}$ Consequently, the needle insertion point and orientation in the skin periphery are crucial to successfully intercept the puncture target.

The optimal patient position and puncture site for accessing the collecting system remain controversial issues. The safety and efficacy of different surgical positions, mainly prone and supine ones, have lately been compared in several studies. ${ }^{40-44}$ Usually, the patient is in the supine position at the start of surgery for performance of a retrograde study and is subsequently repositioned in the prone position for the remaining phases. Because patient reposition increases surgery time of about 30 to 40 minutes, however, some practitioners opt to perform the entire procedure with the patient in the supine position. ${ }^{25,45}$

The prone position in PCNL is frequently associated with patient discomfort, especially for those with severe musculoskeletal deformities, cardiovascular and respiratory problems. ${ }^{11}$ Despite its shortcomings, the prone position facilitates the puncture stage by avoiding abdominal visceral injuries and allowing posterior access to the collecting system, with no limits for instrument excursion and multiple accesses.

Although the supine position has been described as more attractive, being associated with improved levels of comfort for both patient and surgeon, it is limited for upper-pole caliceal puncture, because of its medial, posterior, and concealed position in the rib cage. The use of a real-time 3D trajectory to guide the surgeon throughout the puncture path may increase the viability of performing the entire surgery with the patient in the supine position.

A new surgical tool have also been reported. Yan and coworkers ${ }^{46}$ developed a needle with a system of piezoelectric crystals that allows the needle to be adjusted for particular orientations, which permits motion compensation. This study evaluated different factors such as the input tension and piezoelectric crystals length and thickness to produce particular needle deflections. Other needles integrate a bioimpedance at the tip, which decrease in the resistivity from 1.9 to $1.1 \Omega$ at the renal calix entrance ${ }^{10}$ or electromagnetic sensors that provide real-time information of position and orientation (5 DOFs degrees of freedom) with millimetric precision. ${ }^{19}$ These needles with electromagnetic sensors are related to navigation systems-eg, the system Aurora (Northern Digital Inc., Waterloo)—which sensors are capable of being sterilized and used at medical level.

Besides these types of needles that incorporate sensors and actuators, allowing real-time path compensation and providing information concerning the position and orientation of the needle tip, mathematical models have also been reported to provide a contribution in helping the puncture stage. Usually these necessitate previous quantification of biomechanical properties of tissues, material, and geometry of the needle and insertion properties (speed, strength and orientation). ${ }^{21,47}$

Hing and associates ${ }^{20}$ described a finite element model to simulate needle insertion and removal in soft tissues, at speeds of $1.016 \mathrm{~mm} / \mathrm{s}, 12.7 \mathrm{mms} / \mathrm{s}$, and $24.4 \mathrm{~mm} / \mathrm{s}$. It was found that the lower the insertion speed, the lower was the force exerted on the needle tip and the deformation of soft tissues.

Medical instrumentation-present and future. Several surgical and technological paths have been explored to facilitate and improve the different stages of PCNL. Thus, new surgical positions, simulation systems for puncture training instrumented needles, and biomechanical models can contribute to puncture effectiveness, decrease the number of attempts and procedure time.

Consequently, the development of new surgical instruments, biocompatible with imaging systems, easy to use, durable, and capable of transmitting force and movement haptic feedback (with tremors compensation), may greatly facilitate many of the PCNL steps as well as other MIS procedures. Moreover, needles that allow the spatial monitoring of the tip can be used to reduce respiratory movements and tissues deformations influences. 


\section{Medical robotic for PCNL}

During the last decade, robot-assisted surgery has become an increasingly popular approach to the management of a variety of urologic disorders. Several robots with different levels of specialization, precision, and automation have emerged with high potential to improve surgical performance by providing precise and stable movements and decreasing surgeon dependence. ${ }^{17,18,48}$ Currently, most of these systems are based on medical imaging information, because it is decisive for all PCNL stages.

Among the various robotic devices reported, only a few may be specialized to a level that allows the improvement of the puncturing step in PCNL.

PAKY-RCM, ${ }^{18}$ reported by the Johns Hopkins University group (Baltimore, MD), was developed to aid the PCNL step puncture. This system supports a needle driven by a robotic arm (7 DOFs) and an actuator, allowing the needle positioning in the skin puncturing site and execution of its insertion, using rotational movements, and quantifying the strength exercised at any time of puncture. The surgeon, guided by fluoroscopic images, controls all movements of the robot via a joystick. Experimental tests in humans reported errors of $2 \mathrm{~mm}$ and a nonsignificant difference between the procedure performed by PAKY-RCM and the manual procedure (10.4 \pm 6.5 vs $15.1 \pm 8.8, P=0.06)$. Similar to PAKY-RCM, the AcuBot robot adds a bridge support over the table of the imager and a linear prepositioning stage with 3 DOFs. ${ }^{18}$

Given the MR advantages over fluoroscopy, especially the absence of ionizing radiation, the same group reported the Johns Hopkin MrBot, ${ }^{18}$ which employs a new generation of pneumatic stepper motors using nonmagnetic and dielectric materials. This system, with 6 DOFs, has a great potential for PCNL, given its demonstrated precision of $0.72 \pm 0.36 \mathrm{~mm}$ in in vitro assays.

Lately, dozens of research works have been reported concerning the development and testing of US-guided robotic systems. ${ }^{48}$ The typical approach resorts to a surgical needle attached to a robotic arm that is driven-automatically or controlled by the surgeon-in a 3D or 2D imaging volume. In the $3 \mathrm{D}$ case, the imaging volume results from the acquisition of a 3D US or by overlapping multiple 2D US images, taking into account information from tracking systems, image processing algorithms, and computer graphics. The robotic arms, whose control is automatic, are also represented in volume imaging, using similar navigation systems.

Although recent studies do not aim at conducting a surgical needle in PCNL, live biopsy procedures have been reported with an accuracy of approximately 2 to $6 \mathrm{~mm}$. Recent studies also present the use of robotic arms to support and compensate gravity during needle insertion. A locator apparatus that stabilizes the needle during the puncture was tested in the study by Lazarus and Williams, ${ }^{14}$ in which the authors achieved a mean puncture time of 225 seconds for kidney access, which puncture time is much quicker than the average puncture one reported in the literature (approximately $12 \mathrm{~min}) .{ }^{11,49}$ The robotic arm DLR MIRO with 7 DOFs, $10 \mathrm{Kg}$, and $30 \mathrm{~N}$ of strength has been introduced by Hagn and colleagues. ${ }^{50}$ Laboratory tests, exploring its medical surgery suitability, demonstrated its ability for aligning endoscopes, biopsy needles, electrodes, and brain stimulation catheters, with millimetric precision.
Medical robotics-present and future. Considering the state of the art, one may conclude that surgeons are still in the early stages of understanding and taking advantage of the full potential of robots in medical practice. One should note, however, that robot introduction in surgery procedures is increasing steadily, mainly because of the potential of these systems to increase surgeon movement accuracy and the overall quality of surgeries. Consequently, the number of urologists with training in robot-assisted surgery have increased. $^{13}$

Medical robotics also constitutes an essential component in the path for achieving full functional telesurgery, a concept that has been widely debated in the literature, ${ }^{17,18,48}$ with many associated advantages — of which, for the study, the most relevant one is the elimination of surgeon radiation exposition.

Despite its unquestionable advantages, medical robotics is still struggling to overcome some important problems regarding its widespread use, in particular the difficult initial setups, expensive costs, mechanical problems, absence of tactile feedback, and steep learning curves associated with the acquisition of proficiency.

New developments in medical robotics should focus on improving these disadvantages as well as on the easy attachment of surgical instruments, sterilization and installation facilitation, security, and more independence of imaging systems.

\section{Computer-aided surgery for PCNL}

The combination of medical imagiology and tracking systems has recently been used for computer-assisted navigation systems. Different works have been proposed in the literature regarding navigation software and augmented reality systems. Most of them start by emphasizing the target structures of surgery and surrounding tissues in preoperative data, using image segmentation algorithms or computer graphics (direct volume or surface rendering). Then, the image processed data are superimposed and registered onto real-time intraoperative video (augmented reality) or static preoperative volume data (navigation software). ${ }^{37}$ The surgical tools are commonly updated using motion tracking systems, such as the Polaris infrared camera or the Aurora electromagnetic system, in real time and with millimetric precision.

Most augmented reality systems have been tested in neurosurgery, otolaryngology, and orthopedics, in which the target organs are assumed to be rigid. ${ }^{37}$ Only a few trials have been applied to urology $y^{51}$; the use of augmented reality in abdominal surgery is more challenging because of tissue deformation and respiratory movements.

Mozer and colleagues ${ }^{39}$ described an optical guided system to superimpose an ultrasound nephrostomy tract onto fluoroscopic images during percutaneous renal access.

Recently, Rassweiler and coworkers ${ }^{5}$ reported an augmented iPad-assisted percutaneous access; before the surgical procedure, all relevant anatomic structures are identified and marked in CT preoperative images. During surgery, the iPad camera is used to obtain intraoperative real-time images and, simultaneously, the monitor shows an enhanced virtual reality of the patient's anatomy. For this purpose, four optical markers, which must always be visible on the iPad screen, were rigidly registered. This system helps the surgeon obtain kidney access by showing the relationships between the surgical structures and surgical instruments. 
Besides PCNL, the number of authors reporting augmented reality systems has increased considerably in the last decade. ${ }^{15,37,51,52}$ Given the similarities between PCNL puncture and the contributions of Teber and associates ${ }^{51}$ and $\mathrm{Su}$ and coworkers ${ }^{52}$ in kidney access using image navigation systems for laparoscopic nephrectomy, it is expected that the use of this approach-and similar ones-provides good opportunities for PCNL improvement.

In addition to augmented reality, Huber and colleagues ${ }^{34}$ reported electromagnetic tracking for navigated renal access in an ex vivo model. The surgical needle is guided from skin puncture site toward the renal calix, according to information retrieved by a catheter that integrates electromagnetic motion tracking sensors. The researchers accomplished shorter puncture times of 14 seconds (after a learning phase of 30 punctures) and with a higher precision of $1.7 \mathrm{~mm}$. Electromagnetic navigation have also been successfully described to guide percutaneous puncture in liver and lungs. ${ }^{23}$

Computer-aided surgery - present and future. Although computer-aided surgery may improve surgical skills of the surgeon, it remains a challenging issue. The lack of nonrigid registration methods that estimate soft organ movements and interaction between surgeon instruments and organs limit its use in medical robotics and multimodal imaging. Therefore, many improvements are still needed for both mathematical algorithms and equipment to reach widespread automated computer-aided surgery in the operating room. Moreover, the registration process concerning pre- and intraoperative images and surgical tools also needs additional medical experts, which can be a constraint in today's clinical environment.

Optical tracking has been very helpful intraoperatively, but optical tracking itself requires preoperatory CT imaging, acquired with the patient lying in the same position as the surgical procedure, and demands a line of view to the optical references that can limit its use in PCNL puncture. Besides respiratory and organ movements, needle deflections may also induce targeting and percutaneous guidance errors.

In contrast, electromagnetic tracking does not depend on a clear line of sight. Therefore, surgical tools with small coils may be placed near the renal stone, acting as a real-time anatomic target locator. Therefore, the sensor may remain associated with the kidney stone, allowing permanent motorization. Then, a needle that also integrates a similar sensor may be used to define and guide the surgeon toward renal stones using a virtual trajectory displayed in a computeraided surgery software.

Although Yaniv and coworkers ${ }^{19}$ concluded that it is difficult to generalize working conditions for each electromagnetic system and small changes in the operating room can improve the system performance because of electromagnetic interferences, they achieved average errors less than $3 \mathrm{~mm}$ in different operating rooms. Consequently, electromagnetic tracking may be a suitable system to perform PCNL puncture.

\section{Conclusion}

The number of PCNL procedures is steadily increasing, despite the fact that the number of trained urologists mastering this technique has not kept up with this growth factor. ${ }^{11}$ This survey specifically focuses on methodologic and technologic advances that may leverage better and easier PCNL puncture.

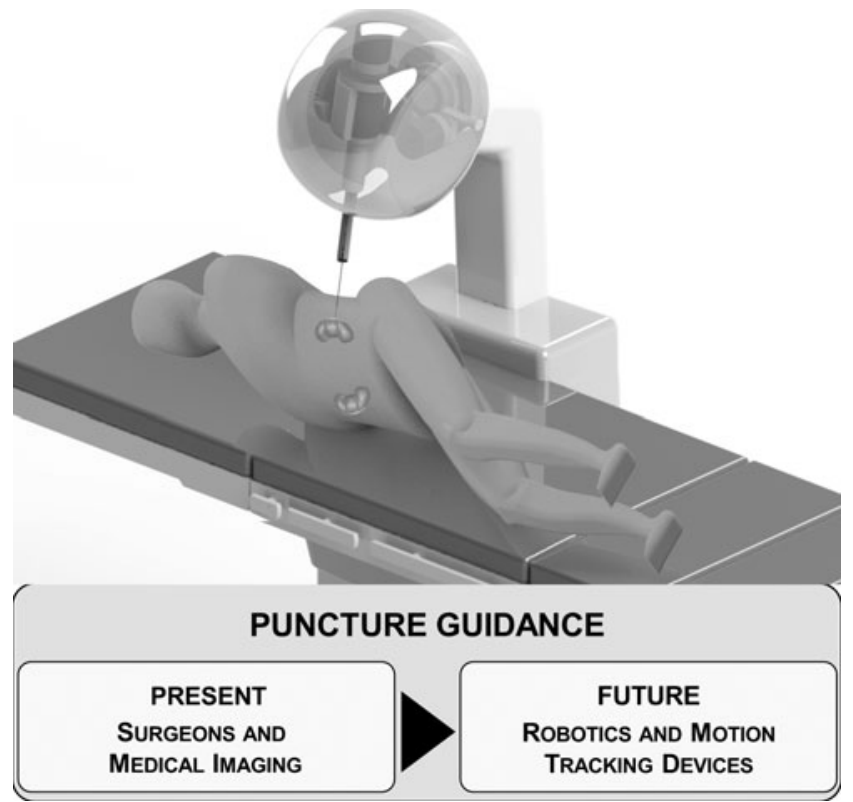

FIG. 2. Present and future developments in percutaneous nephrolithotomy (PCNL) puncture: Regarding PCNL weaknesses (medical imaging limitations, needle deflections, tissue and organs deformations, and surgeon dexterity and precision), robot-assisted surgery and motion tracking devices may be the most accurate and easy way to continuously track the needle tip within the human body using imageless techniques.

Several shortcomings related to image-guided techniques were investigated, showing that real-time tracking and registration of organ motion and deformation remains the biggest challenges to achieve robust navigation. Although breath hold, respiratory gating, and biomechanical models have been used, no widely acceptable solution has yet been reached because of the lack of robustness, precision, and real-time algorithms. Because MR produces high-quality images and eliminates radiation exposure, new ergonomically designed MR systems will certainly constitute an important research path in medical imaging.

Because of the benefits of endoscopic surgery, future developments may focus on camera resolution, field of view and depth perception, tactile feedback, and improvements in mobility and handling of instruments.

Computer-aided surgeries and tracking devices are subject of ongoing research, because they may play an important role in the future of PCNL. These techniques have the potential to provide valuable interfaces between anatomic structures and surgical tools, medical robots, organ deformation, and movements improving puncture planning and guidance. Therefore, navigation systems could represent a step forward to reduce the medical imaging dependence and associated shortcomings in puncture conduction.

It is expected that future improvements in PCNL will comprise the elimination of radiation and reduction of surgical costs and time. Finally, although the development and improvement of autonomous robots may broaden the use of PCNL to surgeons less familiar with MIS, it also raises challenging robustness and safety issues that have to be tackled in the future (Fig. 2). 


\section{Acknowledgment}

The authors acknowledge Foundation for Science and Technology (FCT) for the fellowships references: SFRH/ BPD/46851/2008 and SFRH/BD/74276/2010.

\section{Disclosure Statement}

No conflicts of interest exist.

\section{References}

1. Worcester EM, Coe FL. Calcium kidney stones. N Engl J Med 2010;363:954-963.

2. Armitage JN, Irving SO, Burgess NA. Percutaneous nephrolithotomy in the United Kingdom: Results of a prospective data registry. Eur Urol 2012;61:1188-1893.

3. Knoll T, Buchholz N, Wendt-Nordahl G. Extracorporeal shockwave lithotripsy vs. percutaneous nephrolithotomy vs. flexible ureterorenoscopy for lower-pole stones. Arab J Urol 2012;10:336-341.

4. Sivalingam S, Al-Essawi T, Hosking D. Percutaneous nephrolithotomy with retrograde nephrostomy access: A forgotten technique revisited. J Urol 2012. Epub ahead of print.

5. Rassweiler JJ, Muller M, Fangerau M, et al. iPad-assisted percutaneous access to the kidney using marker-based navigation: Initial clinical experience. Eur Urol 2012;61: 628-631.

6. Michel MS, Trojan L, Rassweiler JJ. Complications in percutaneous nephrolithotomy. Eur Urol 2007;51:899-906.

7. de la Rosette J, Assimos D, Desai M, et al. The Clinical Research Office of the Endourological Society Percutaneous Nephrolithotomy Global Study: Indications, complications, and outcomes in 5803 patients. J Endourol. 2011;25:11-17.

8. Guven S, Istanbulluoglu O, Gul U, et al. Successful percutaneous nephrolithotomy in children: Multicenter study on current status of its use, efficacy and complications using Clavien classification. J Urol 2011;185:1419-1424.

9. Ozden E, Mercimek MN, Yakupoglu YK, et al. Modified Clavien classification in percutaneous nephrolithotomy: Assessment of complications in children. J Urol 2011;185: 264-268.

10. Kalogeropoulou C, Kallidonis P, Liatsikos EN. Imaging in percutaneous nephrolithotomy. J Endourol 2009;23: 1571-1577.

11. de la Rosette JJ, Laguna MP, Rassweiler JJ, Conort P. Training in percutaneous nephrolithotomy-a critical review. Eur Urol 2008;54:994-1001.

12. Cracco CM, Scoffone CM, Scarpa RM. New developments in percutaneous techniques for simple and complex branched renal stones. Curr Opin Urol 2011;21:154-160.

13. Ukimura O. Image-guided surgery in minimally invasive urology. Curr Opin Urol 2010;20:136-140.

14. Lazarus J, Williams J. The Locator: Novel percutaneous nephrolithotomy apparatus to aid collecting system puncture-a preliminary report. J Endourol 2011;25:747-750.

15. Li ZC, Li K, Zhan HL, et al. Augmenting intraoperative ultrasound with preoperative magnetic resonance planning models for percutaneous renal access. Biomed Eng Online 2012;11:60.

16. Bader MJ, Gratzke C, Seitz M, et al. The "all-seeing needle": Initial results of an optical puncture system confirming access in percutaneous nephrolithotomy. Eur Urol 2011;59: 1054-1059.
17. Desai MM, Grover R, Aron M, et al. Robotic flexible ureteroscopy for renal calculi: Initial clinical experience. J Urol 2011;186:563-568.

18. Mozer P, Troccaz J, Stoianovici D. Urologic robots and future directions. Curr Opin Urol 2009;19:114-119.

19. Yaniv Z, Wilson E, Lindisch D, Cleary K. Electromagnetic tracking in the clinical environment. Med Phys 2009;36: 876-892.

20. Hing JT, Brooks AD, Desai JP. A biplanar fluoroscopic approach for the measurement, modeling, and simulation of needle and soft-tissue interaction. Med Image Anal 2007;11: $62-78$.

21. van Gerwen DJ, Dankelman J, van den Dobbelsteen JJ. Needle-tissue interaction forces-a survey of experimental data. Med Eng Phys 2012;34:665-680.

22. Wein W, Brunke S, Khamene A, et al. Automatic CTultrasound registration for diagnostic imaging and imageguided intervention. Med Image Anal 2008;12:577-585.

23. Meyer BC, Peter O, Nagel M, et al. Electromagnetic fieldbased navigation for percutaneous punctures on C-arm CT: Experimental evaluation and clinical application. Eur Radiol 2008;18:2855-2864.

24. Ghani KR, Patel U, Anson K. Computed tomography for percutaneous renal access. J Endourol 2009;23:1633-1639.

25. Miller J, Durack JC, Sorensen MD, Wang JH, Stoller ML. Renal calyceal anatomy characterization with 3-dimensional in vivo computerized tomography imaging. J Urol 2013;189: 562-567.

26. Ritter M, Rassweiler MC, Häcker A, Michel M. Laser-guided percutaneous kidney access with the Uro Dyna-CT: First experience of three-dimensional puncture planning with an ex vivo model. World J Urol 2012. Epub ahead of print.

27. Sorensen MD, Harper JD, Hsi RS, et al. B-mode ultrasound versus color Doppler twinkling artifact in detecting kidney stones. J Endourol 2013;27:149-153.

28. Jessen JP, Honeck P, Knoll T, Wendt-Nordahl G. Percutaneous nephrolithotomy under combined sonographic/ radiologic guided puncture: Results of a learning curve using the modified Clavien grading system. World J Urol 2013. Epub ahead of print.

29. Leroy A, Mozer P, Payan Y, Troccaz J. Rigid registration of freehand 3D ultrasound and CT-scan kidney images. Medical Image Computing and Computer-Assisted Intervention MICCAI 2004, Pt 1, Proc Int Conf 2004;3216:837-844.

30. Mozer P, Leroy A, Payan Y, et al. Computer-assisted access to the kidney. Int J Med Robot 2005;1:58-66.

31. Markelj P, Tomazevic D, Likar B, Pernus F. A review of 3D/ $2 \mathrm{D}$ registration methods for image-guided interventions. Med Image Anal 2012;16:642-661.

32. Desai MR, Sharma R, Mishra S, et al. Single-step percutaneous nephrolithotomy (microperc): The Initial clinical report. J Urol 2011;186:140-145.

33. Colangelo CJ, Kaplan G, Palazzi K, et al. Ureteroscopy in pediatric patients with spinal abnormalities. J Endourol 2013. Epub ahead of print.

34. Huber J, Wegner I, Meinzer HP, et al. Navigated renal access using electromagnetic tracking: An initial experience. Surg Endosc 2011;25:1307-1312.

35. Mancini JG, Raymundo EM, Lipkin M, et al. Factors affecting patient radiation exposure during percutaneous nephrolithotomy. J Urol 2010;184:2373-2377.

36. Cleary K, Peters TM. Image-guided interventions: Technology review and clinical applications. Annu Rev Biomed Eng. 2010;12:119-142. 
37. Nicolau S, Soler L, Mutter D, Marescaux J. Augmented reality in laparoscopic surgical oncology. Surg Oncol 2011;20: 189-201.

38. Mishra S, Jagtap J, Sabnis RB, Desai MR. Training in percutaneous nephrolithotomy. Curr Opin Urol 2013;23: 147-151.

39. Mozer P, Conort $\mathrm{P}$, Leroy A, et al. Aid to percutaneous renal access by virtual projection of the ultrasound puncture tract onto fluoroscopic images. J Endourol 2007;21:460-465.

40. Autorino R, Giannarini G. Prone or supine: Is this the question? Eur Urol 2008;54:1216-1218.

41. de la Rosette JJ, Tsakiris P, Ferrandino MN, et al. Beyond prone position in percutaneous nephrolithotomy: A comprehensive review. Eur Urol 2008;54:1262-1269.

42. De Sio M, Autorino R, Quarto G, et al. Modified supine versus prone position in percutaneous nephrolithotomy for renal stones treatable with a single percutaneous access: A prospective randomized trial. Eur Urol 2008;54:196-202.

43. Karami H, Mohammadi R, Lotfi B. A study on comparative outcomes of percutaneous nephrolithotomy in prone, supine, and flank positions. World J Urol 2012. Epub ahead of print.

44. Honey RJ, Wiesenthal JD, Ghiculete D, et al. Comparison of supracostal versus infracostal percutaneous nephrolithotomy using the novel prone-flexed patient position. J Endourol. 2011;25:947-954.

45. DasGupta R, Patel A. Percutaneous nephrolithotomy: Does position matter?- - prone, supine and variations. Curr Opin Urol 2013;23:164-168. 10.1097/MOU.0b013e32835d3067.

46. Yan KG, Podder T, Yu Y, et al. 'Smart' needle for percutaneous surgery: Influential factor investigation. Conf Proc IEEE Eng Med Biol Soc 2007;2007:461-464. PubMed PMID: ISI:000253467000116. English.

47. DiMaio SP, Salcudean SE. Needle steering and motion planning in soft tissues. IEEE Trans BioMed Eng 2005;52: 965-974.

48. Cinquin P. How today's robots work and perspectives for the future. J Visc Surg 2011;148:E12-E18.
49. Li X, Liao S, Yu Y, et al. Stereotactic localisation system: A modified puncture technique for percutaneous nephrolithotomy. Urol Res 2012;40:395-401.

50. Hagn U, Nickl M, Jorg S, et al. The DLR MIRO: A versatile lightweight robot for surgical applications. Ind Robot 2008; 35:324-336.

51. Teber D, Guven S, Simpfendörfer T, et al. Augmented reality: A new tool to improve surgical accuracy during laparoscopic partial nephrectomy? Preliminary in vitro and in vivo results. Eur Urol 2009;56:332-338.

52. Su LM, Vagvolgyi BP, Agarwal R, et al. Augmented reality during robot-assisted laparoscopic partial nephrectomy: Toward real-time 3D-CT to stereoscopic video registration. Urology 2009;73:896-900.

Address correspondence to: Pedro L. Rodrigues, MSc

Life and Health Sciences Research Institute (ICVS) School of Health Sciences

University of Minho 4710-057 Braga Portugal

E-mail: pedrorodrigues@ecsaude.uminho.pt

$\begin{aligned} & \text { Abbreviations Used } \\ \mathrm{CT} & =\text { computed tomography } \\ \mathrm{DOF} & =\text { degrees of freedom } \\ \mathrm{IVU} & =\text { intravenous urography } \\ \mathrm{MIS} & =\text { minimally invasive surgery } \\ \mathrm{MR} & =\text { magnetic resonance } \\ \mathrm{PCNL} & =\text { percutaneous nephrolithotomy } \\ \mathrm{SWL} & =\text { shockwave lithotripsy } \\ 3 \mathrm{D} & =\text { three dimensional } \\ 2 \mathrm{D} & =\text { two dimensional } \\ \mathrm{US} & =\text { ultrasonography }\end{aligned}$

